\title{
Study on the Influence of Geometric Shape of Material on the Reposed Angle Based on EDEM
}

\author{
Xuan Lin, Yanhui Chen* \\ School of Mechanical Engineering, Guangxi University of Science and Technology, Liuzhou, China \\ Email address: \\ 824777965@qq.com (Xuan Lin), gxut_jx@163.com (Yanhui Chen) \\ ${ }^{*}$ Corresponding author
}

To cite this article:

Xuan Lin, Yanhui Chen. Study on the Influence of Geometric Shape of Material on the Reposed Angle Based on EDEM. International Journal of Mechanical Engineering and Applications. Vol. 6, No. 4, 2018, pp. 110-125. doi: 10.11648/j.ijmea.20180604.14

Received: September 28, 2018; Accepted: October 15, 2018; Published: October 18, 2018

\begin{abstract}
When studying the discreteness of granular materials, the size of the angle of repose can be used as an important parameter to study the stability of granular materials. In recent years, many scholars have studied the morphology of particles, but they are basically based on the regular shape of spheres and ellipsoids, which are very different from the actual particle shape, which leads to the measurement results not consistent with the actual results. Therefore, based on the above reasons, this paper mainly studied the influence of geometric shape for the Reposed Angle, the simulation experiment is carried for the Reposed Angle using the discrete element software EDEM, the Reposed Angle is measured for five different shape using slice cutting analysis method, and the experimental measurement has carried on for the Reposed Angle for each shape, SPC method is used to determine the reliability of the data. The results show that the size of the Reposed Angle increases gradually in the order of sheet, intermediate, horn, strip and equal square among the five studied shape, the mechanism is that the greater the number of internal contact is, can truly reflect the interaction and occlusal relationship, the more easily locked the particles are in the force chain, the more difficult to separate the particles are, the more stable the accumulation characteristics are.
\end{abstract}

Keywords: Geometric Shape, The Reposed Angle, EDEM, SPC

\section{Introduction}

Granular material is a kind of loose material, its discrete properties make it different from other physical media [1-2]. The Reposed Angle is the basic macro parameter to reflect the discrete characteristics of particles, it can be used as an indicator for the dispersion and accumulation of materials. However, it is often associated with the collapse, segregation and stratification of materials during the process of the measurement of the Reposed Angle, the importance of the Reposed Angle gradually has became known. Wang jianguo [3] studied the influence of different accumulation modes on the size of Reposed Angle by using Discrete Element Method naturally. Li changing [4] established the mathematical model between the Reposed Angle and particle size and non-uniformity through numerical simulation analysis, studying size of the Reposed Angle. Li Yanjie [5] used the discrete element method to study the important parameters affecting the size of the angle of repose. Through the combination of experimental results and numerical simulation, it is pointed out that the discrete element method has certain applicability and feasibility in studying the particle accumulation problem. Sexual and scientific. From the above, Discrete Element Method is feasible and scientific in studying particle accumulation when studying the relevant properties of granular materials, the Reposed Angle can be used as an important index to study the properties of granular materials.

Many scholars have studied particle morphology in recent years, but it's based on the shape of the spheroid and ellipsoid basically, it is very different from the actual particle shape, the result of measurement does not agree with the actual result. It's simple for angular grain shapes, particle shapes vary widely in nature, can't do numerical all simulations. In this paper, several representative rock particle shapes are selected to measure the size of the Reposed Angle, the mechanical properties and stability characteristics of granular materials are studied. 


\section{The Basic Principle of Discrete Element Method and EDEM Software Introduction}

\subsection{The Basic Principle of Discrete Element Method}

Discrete Element Method is a numerical simulation method for solving discrete media problems. The principle is to divide the research objects into independent units with mutual contact. Cyclic iterative calculation is performed through dynamic relaxation method or static relaxation method based on Newton's laws of motion, making sure that the force of all units can be balanced in small enough time. And always update the displacement position of all units, the micro motion of each unit is tracked and calculated, so that perform macroscopic motion law of the whole research object [6].

The discrete element method mainly involves two major contents, the contact model and Newton's law of motion. Among them, the contact model is mainly used to calculate the contact force between each discrete unit, and Newton's law of motion is used to calculate the displacement, velocity and acceleration of each discrete unit. Since the discrete element method is based on different contact models and Newton's law of motion, its research objects are diversified and the research problems are complicated. Therefore, the contact models and algorithms used by the discrete element method are also different. For discrete particle systems, a single discrete particle is treated as a single unit; but for a rock particle system, a single polygonal block is treated as a single unit.

\subsection{EDEM Software Introduction}

EDEM software is a CAE software for simulating and analyzing bulk materials developed by DEM-solutions UK based on discrete element method [7]. With the development of industrial development requirements and the development of computing technology, EDEM has been widely used in many fields. EDEM software is similar to other CAE software, and usually has three modules: pre-processing, solution, and post-processing. Its pre-processing has the function of creating and initializing models. In this module, it mainly performs tasks such as particle creation, model definition, particle and component input. The solution module mainly performs setting of various options, simulation execution and simulation. Viewing of the process; subsequent processing modules provide tools for analyzing and judging the simulation data and their extended application functions.

\section{Establishment of Simulation Model of Bottomless Cylinder Test}

\subsection{Selection of Contact Model and Setting of Contact Parameters}

In particle contact theory, contact model is an important basis of Discrete Element Method. There are specific contact models for different simulation objects. The hertz-mindlin non-slip contact model is selected to calculate the force between particles [8] because the object of the study is gravel in EDEM. It is necessary to set the correct parameters of the simulation model. The property parameters of the relevant materials are shown in Table 1 and Table 2 [9-10].

Table 1. The property parameter table of the material.

\begin{tabular}{llll}
\hline material & poisson's ratio & Shear modulus $(\mathbf{p a})$ & Density $\left(\mathbf{k g} / \mathbf{m}^{\mathbf{3}}\right)$ \\
\hline gravel & 0.20 & $5 \mathrm{e}+07$ & 2600 \\
steel & 0.30 & $7 \mathrm{e}+10$ & 7800 \\
\hline
\end{tabular}

Table 2. Material's contact attribute parameter table.

\begin{tabular}{llll}
\hline material & coefficient of restitution & coefficient of static friction & Coefficient of rolling friction \\
\hline gravel-gravel & 0.5 & 0.9 & 0.15 \\
gravel-steel & 0.5 & 0.4 & 0.05 \\
\hline
\end{tabular}

\subsection{The Establishment of the Particle Geometry Model}

In reality, the shapes of crushed stone particles vary widely. Particles are usually subdivided into strip, horn, equal square, sheet, intermediate type for five types [11]. In the past, some scholars used reversed engineering to create the $\mathrm{CAD}$ model of gravel particles, you can get the outer contour of the actual crushed stone, it have high accuracy, five particle models are created respectively in the EDEM software, As shown in Figure 1. 


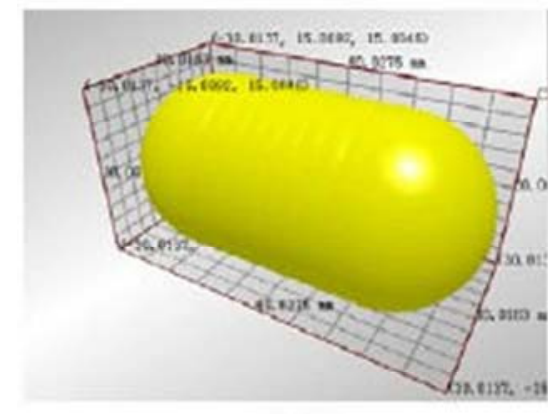

1. Strip

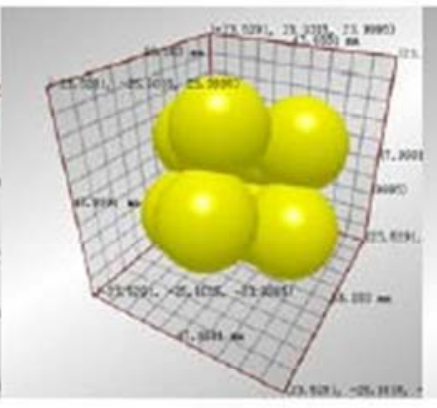

2. Equal Square

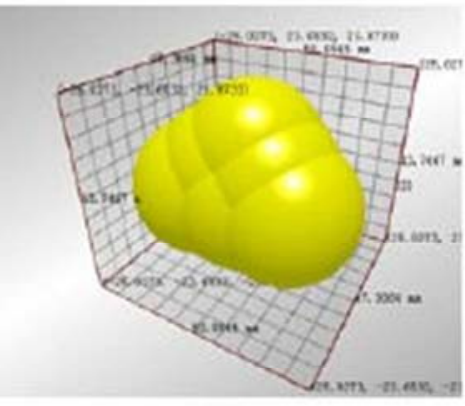

3. Horn

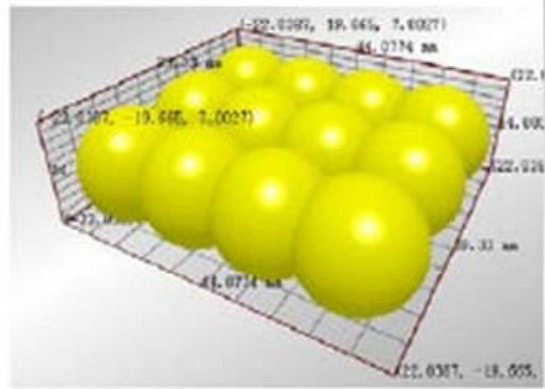

4. Sheet

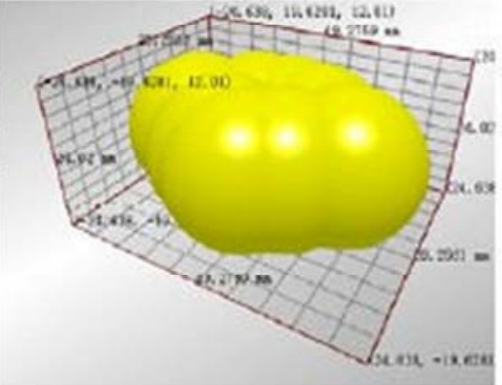

5. Intermediate

Figure 1. Five gravel model type of rock material.

\subsection{Establishment of Simulation Model of Bottomless Cylinder}

The simulation model of the bottomless cylinder is composed of four basic processes, including the modeling of the bottomless cylinder, the filling of gravel particles, the outflow of gravel particles and the natural accumulation of crushed stone particles, As shown in Figure 2. The tool of the model consists of a cylinder and a liner, the diameter and height of the bottomless cylinder are $225 \mathrm{~mm}$ and $675 \mathrm{~mm}$ respectively. In order to ensure the integrity of the material structure and the normal edge diffusion, the lifting speed proposed is setted up for $0.05 \mathrm{~m} / \mathrm{s}$ by li qinliang [12], 5 simulation experiments are conducted.

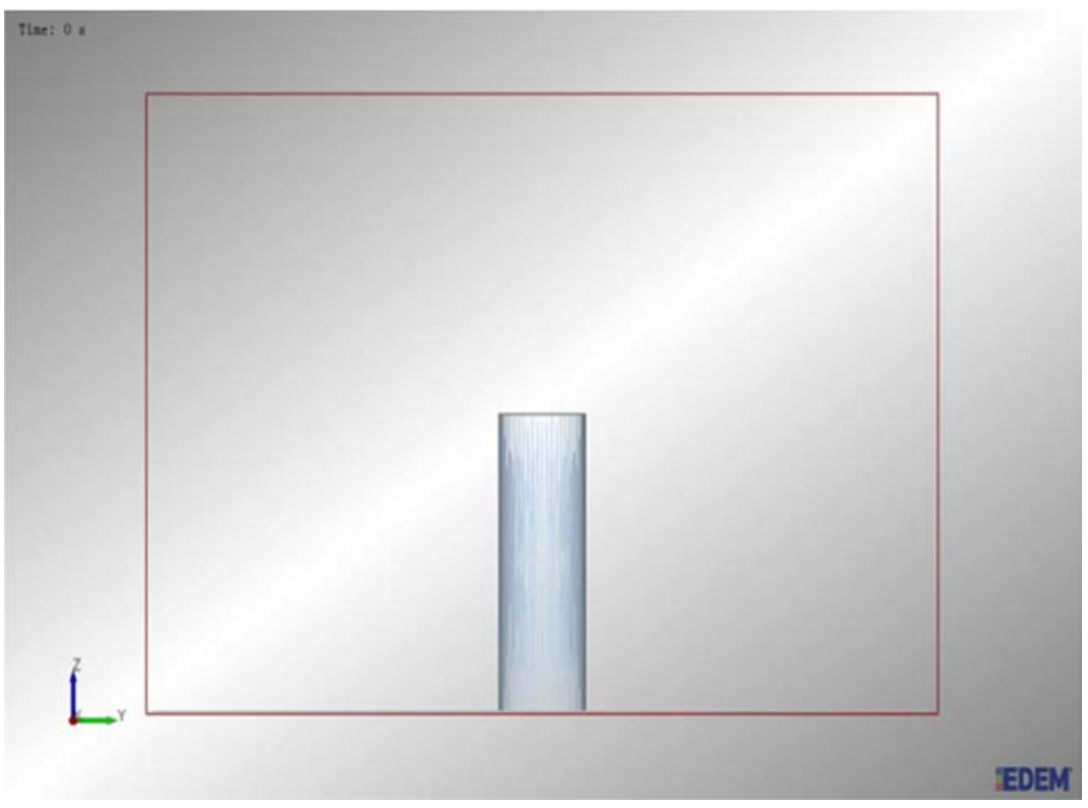

(a) The modeling of the bottomless cylinder 


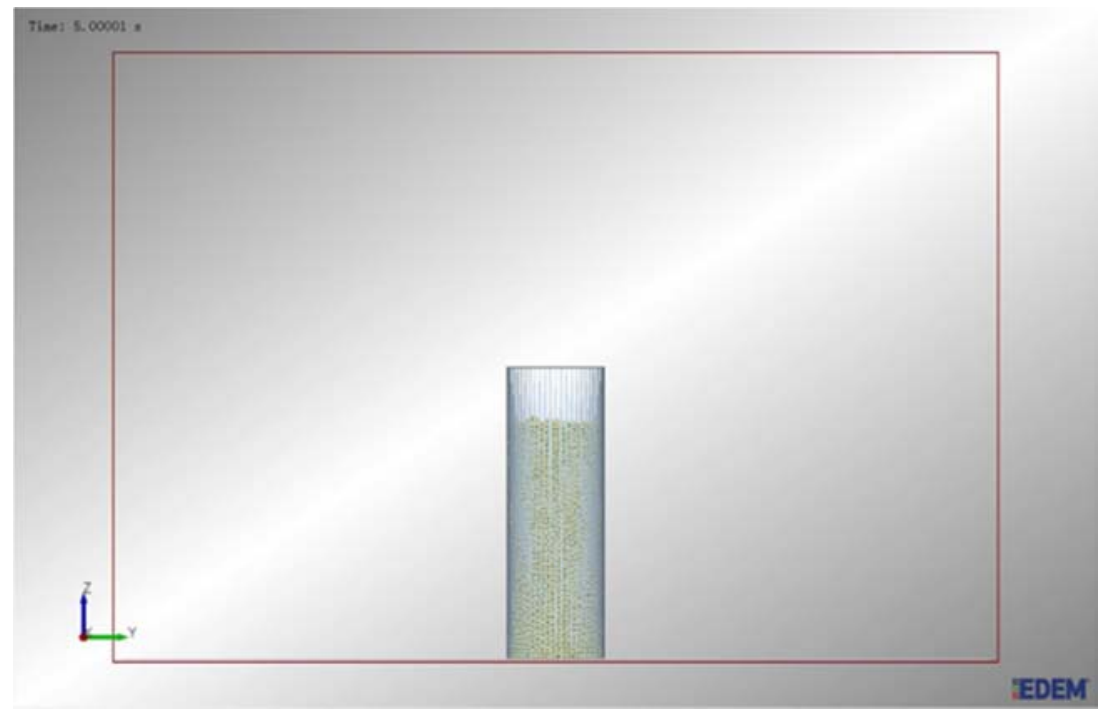

(b) The filling of gravel particles

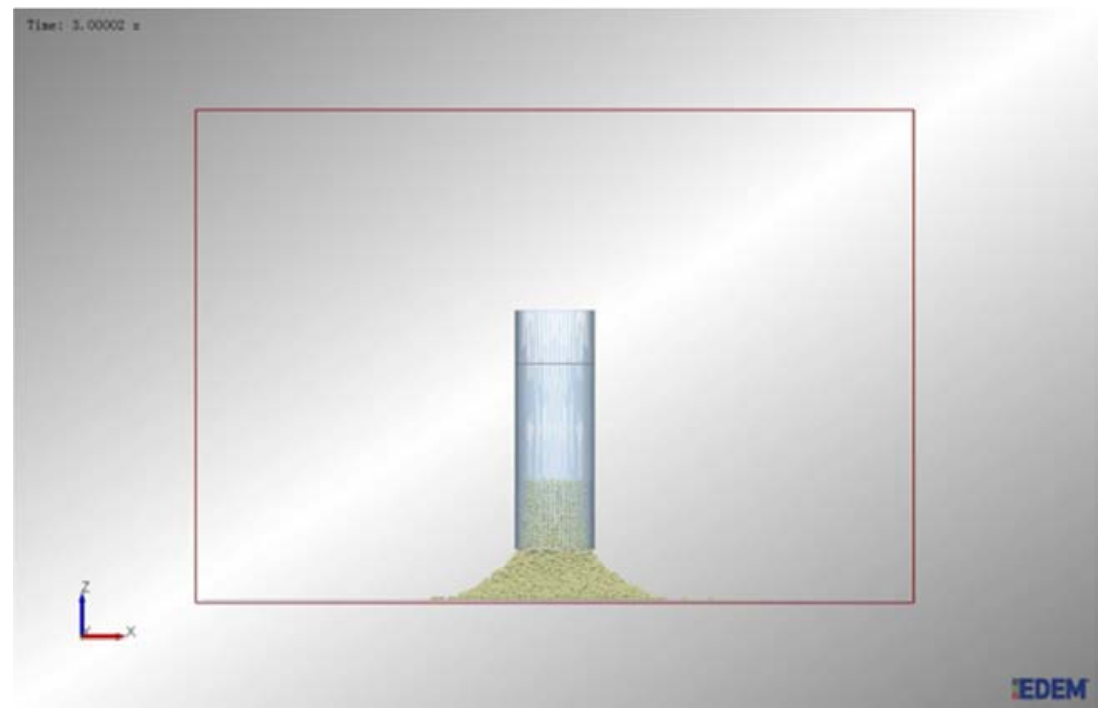

(c) The outflow of gravel particles

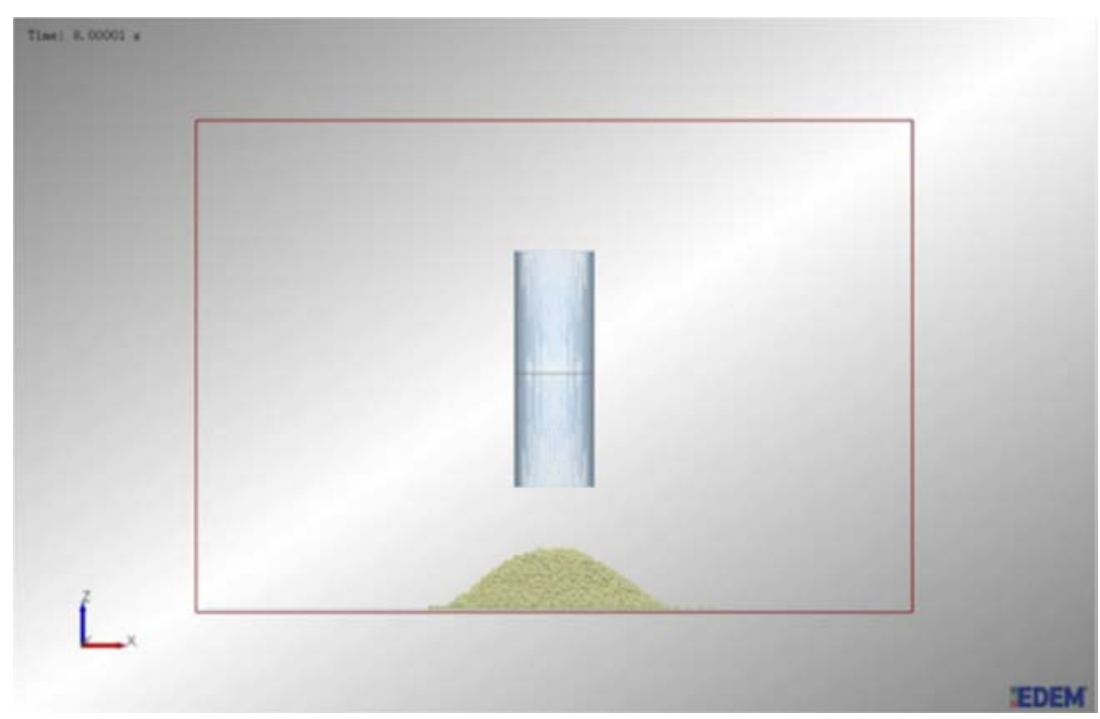

(d) The natural accumulation of crushed stone particles

Figure 2. The process of Simulation Model of bottomless cylinder 


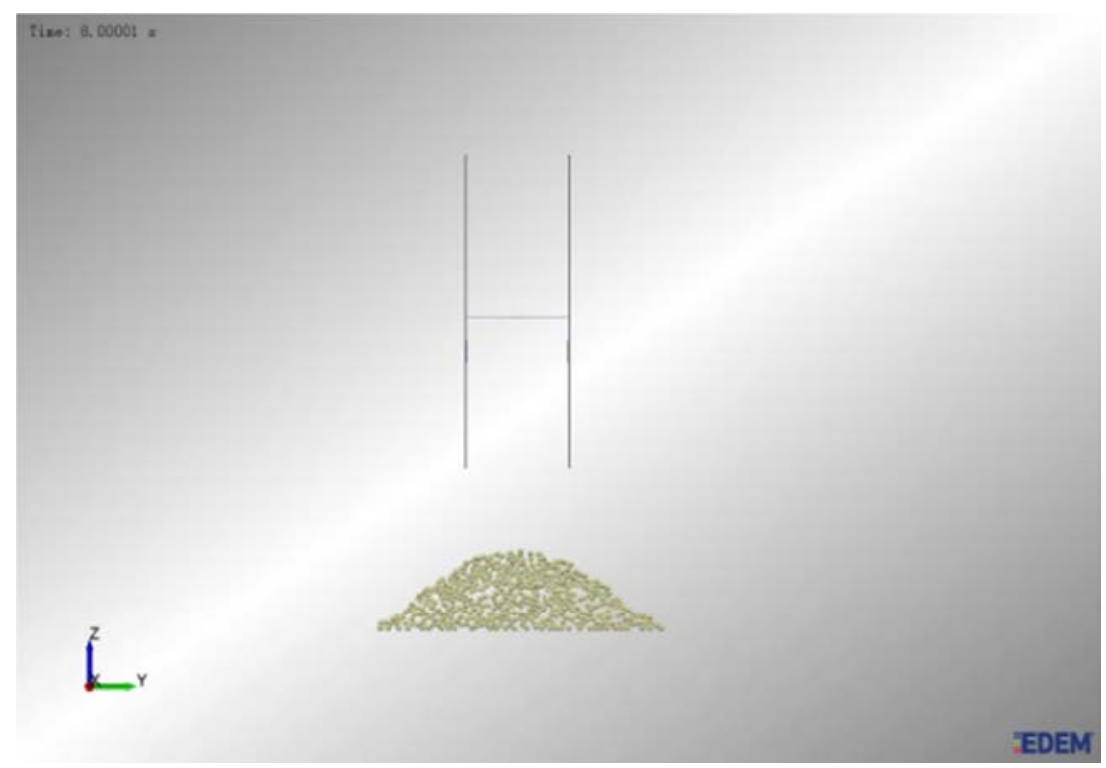

Figure 3. The truncation diagram of xoz section

In order to ensure the accuracy of the Reposed Angle, the slice processing function is used to slice and treat 5 groups of different shape granules in EDEM software. Cut off the appropriate thickness of the particle stack xoz plane, the center coordinates of the most epitaxial particles are derived on the left and right sides of each section. Then doing a fitting linear and measuring the Reposed Angle on both sides, the average is the size of the Reposed Angle, the sheet slice truncation is shown in Figure 3, the same is true for other shapes.

In this paper, the application of excel is performed in the linear fitting of the xoz section of the sheet granule. The linear equation of the Reposed Angle of the left and right sides of the flaps is obtained. Other shapes have the same principle, As shown in Figure 4.

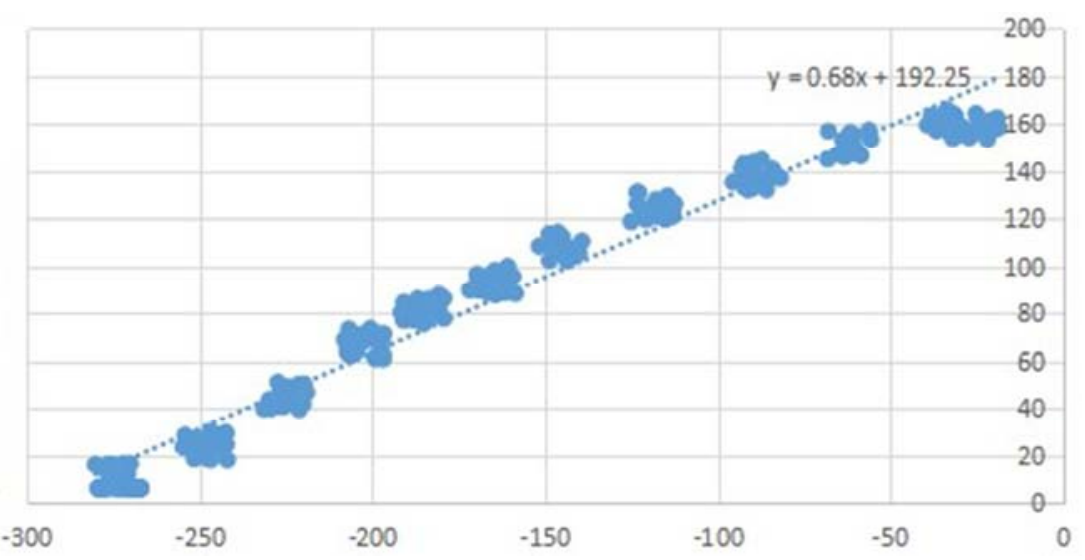

(a) The fitted equation on the left sheet

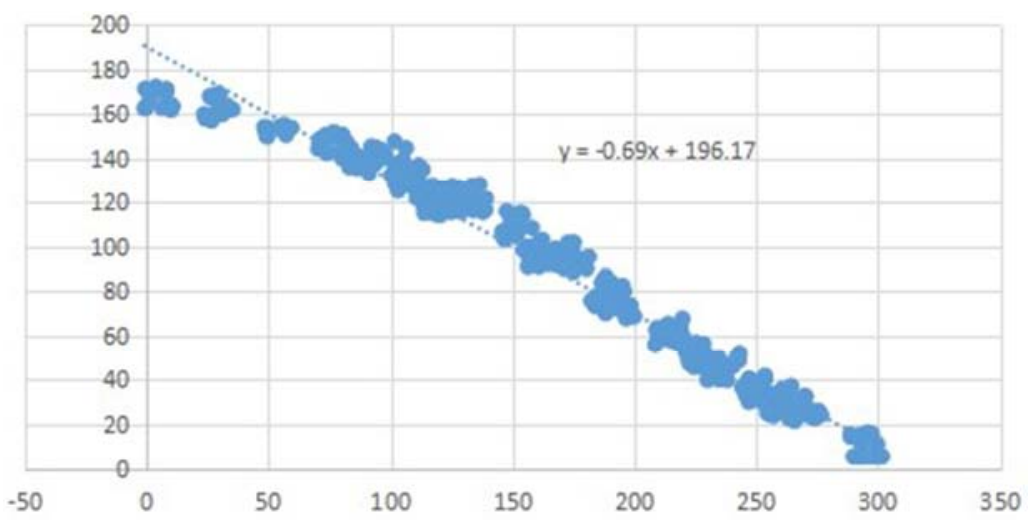

(b) The fitted equation on the right sheet 


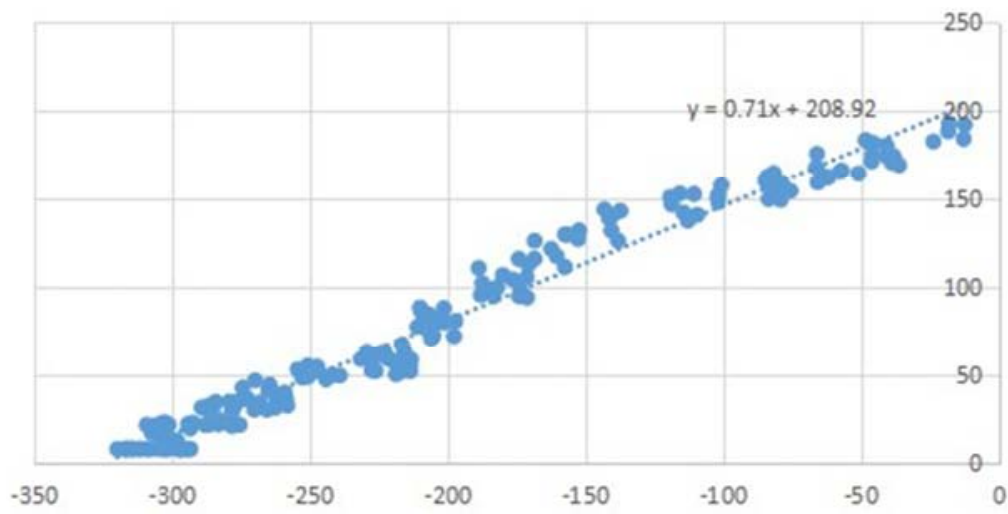

(c) The fitted equation on the left intermediate

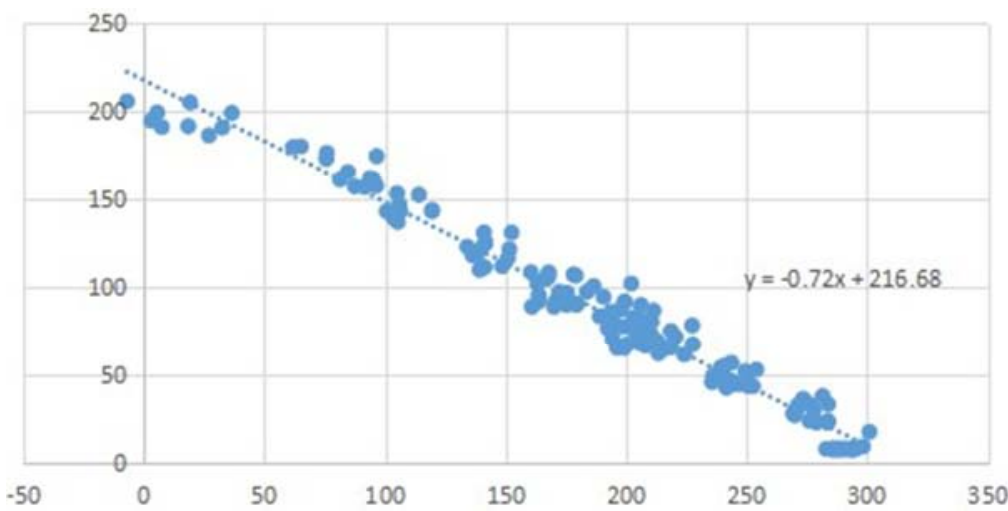

(d) The fitted equation on the right intermediate

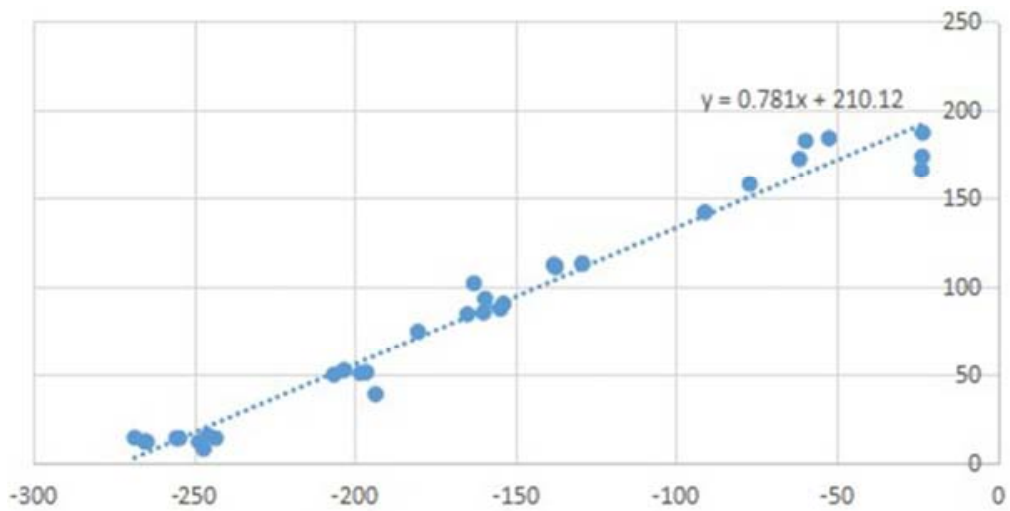

(e) The fitted equation on the left horn

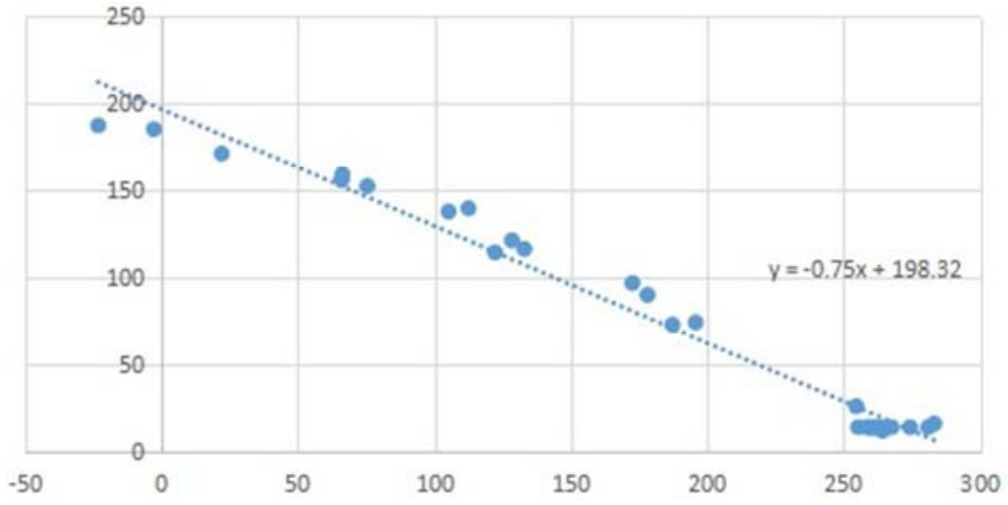

(f) The fitted equation on the right horn 


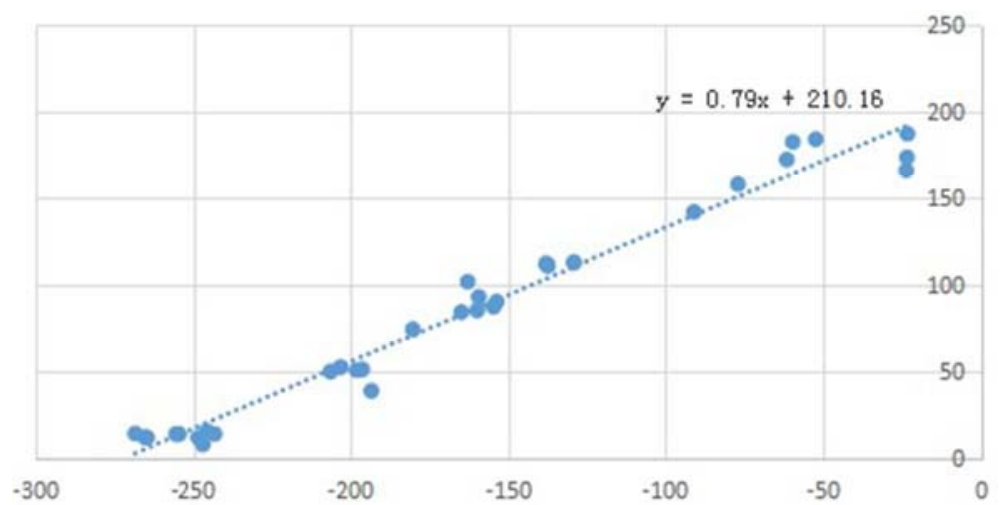

(g) The fitted equation on the left strip

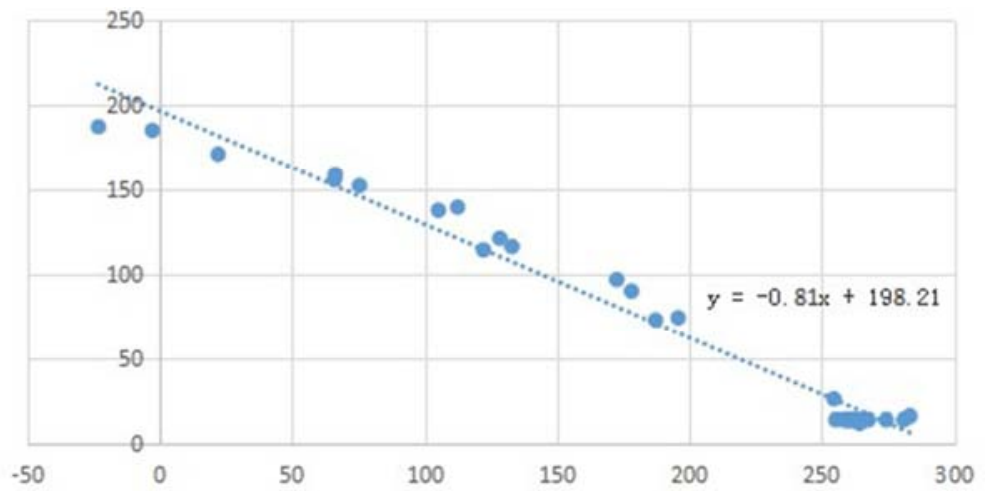

(h) The fitted equation on the right strip

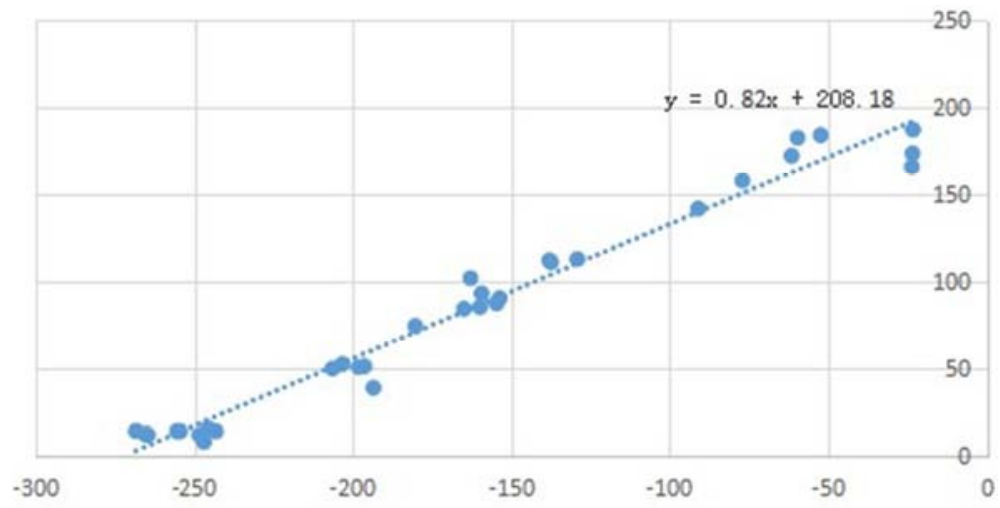

(i) The fitted equation on the left equal square

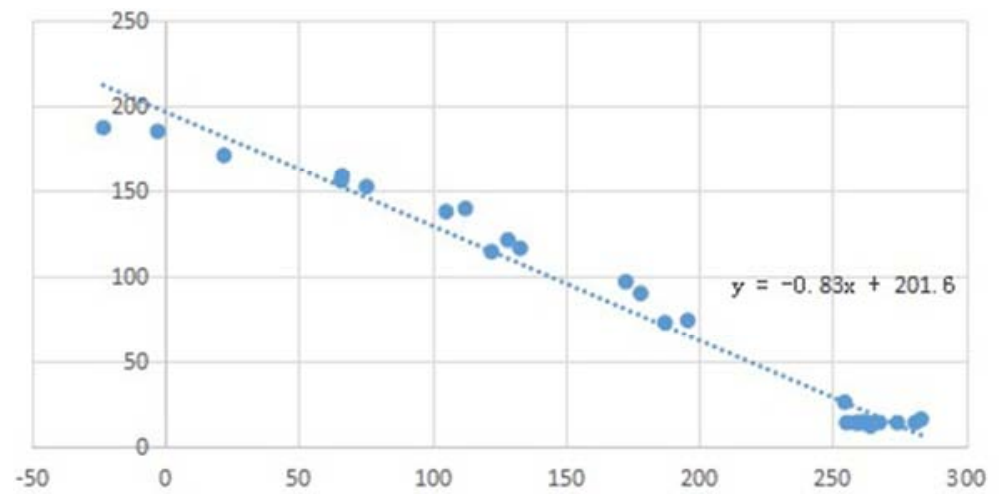

(j) The fitted equation on the right equal square

Figure 4. The left and right fitted Angle of each group of particles. 
As shown in Figure 4, the left and right fitting corresponding Reposed Angle are $34.22^{\circ}$ and $34.61^{\circ}$ respectively, the average is $34.42^{\circ}$. In the same way, you can get the Reposed Angle for groups, As shown in Table 3.

Table 3. The Reposed Angle for Each group.

\begin{tabular}{lllll}
\hline Sheet $\left(^{(}\right)$ & Intermediate $\left(^{(}\right)$ & Horn $\left(^{(}\right)$ & Strip $\left(^{(}\right)$ & Equal square $\left(^{(}\right)$ \\
\hline 34.42 & 35.56 & 37.44 & 38.66 & 39.56 \\
\hline
\end{tabular}

\section{Determination of the Reposed Angle of Particle Material}

The Reposed Angle is similar to the numerical simulation, it is setted for a bottomless cylinder experiment device, the ratio of its diameter to height is $1: 3$ [13], put the corresponding shape particles into the device for each group, the lifted velocity is $0.05 \mathrm{~m} / \mathrm{s}$, the rock material flows from the barrel slowly, after stabilizing the rock particles, forming aggregate model of gravel particles, As shown in Figure 5.

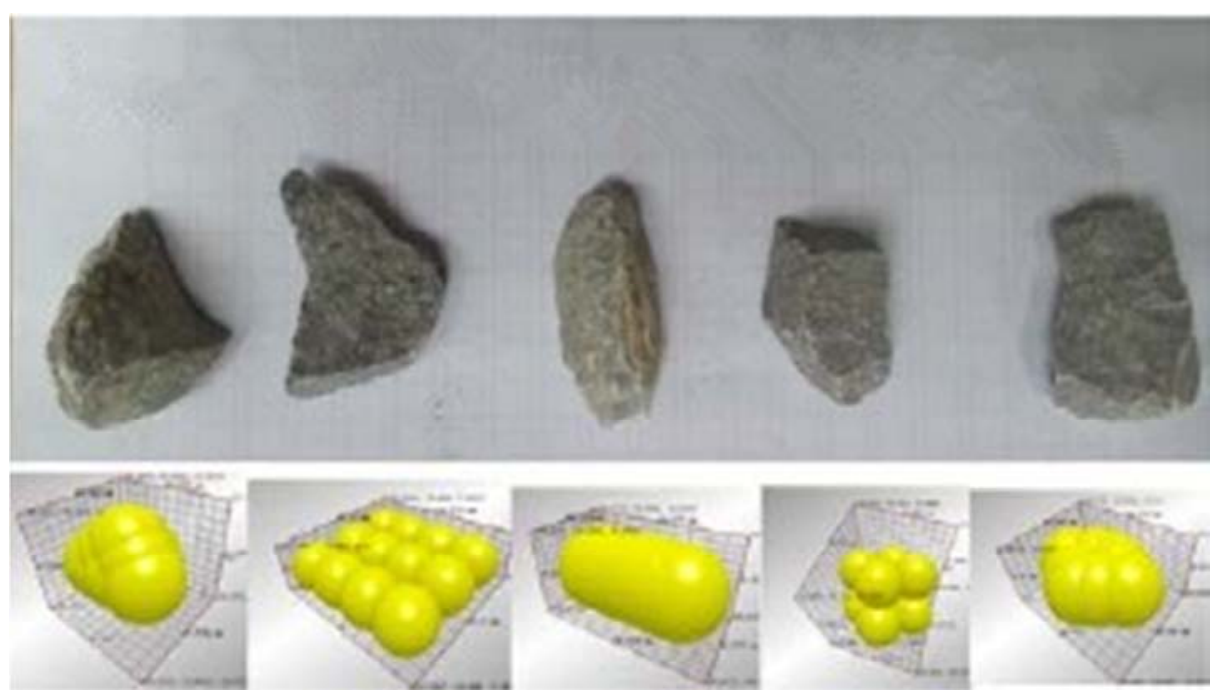

(a) Five kinds of granulated particles

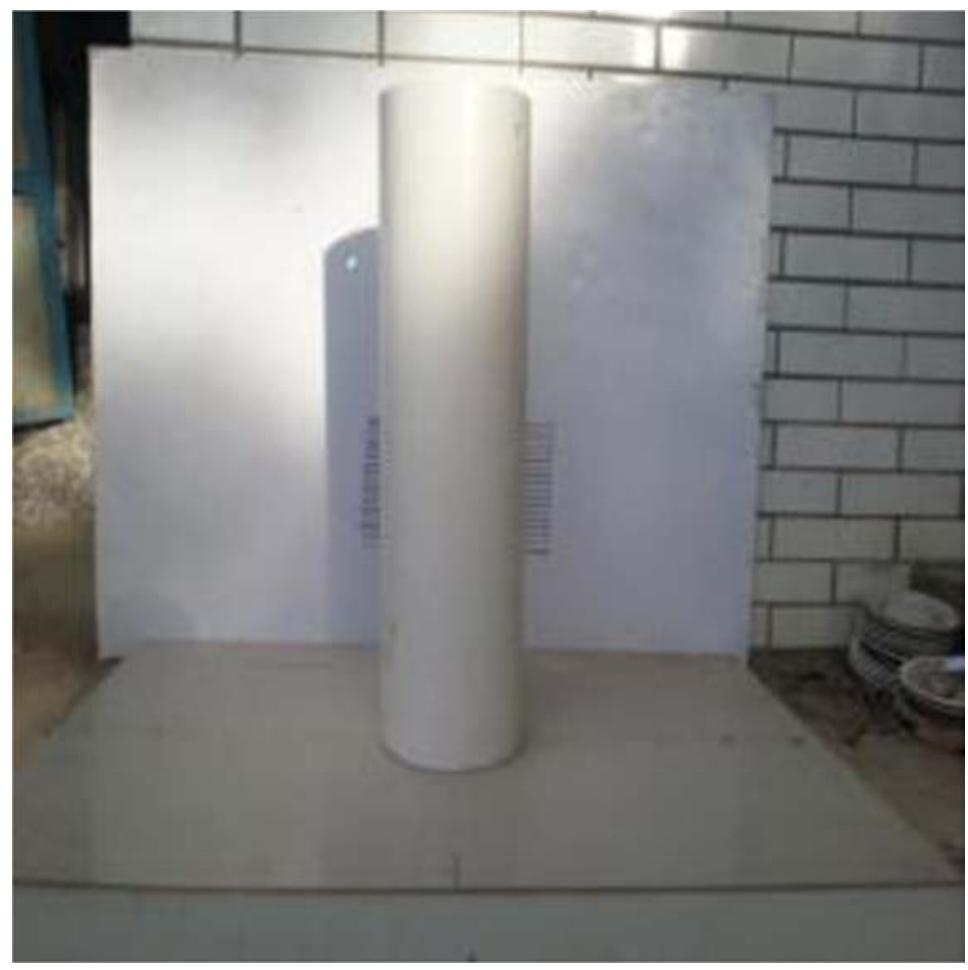

(b) bottomless cylinder experiment device 


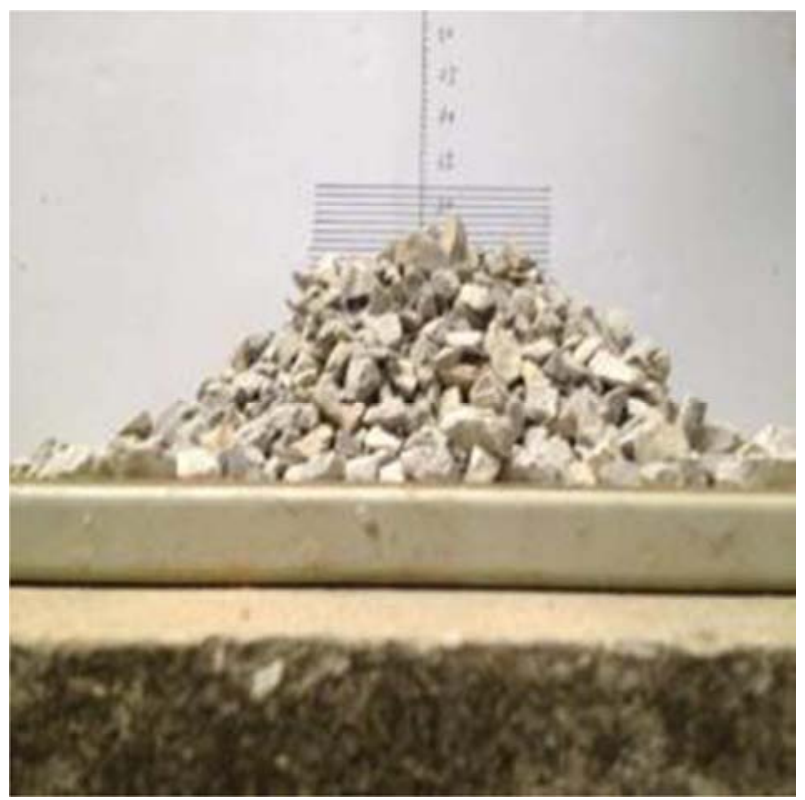

(c) The natural Reposed Angle of the rock particles

Figure 5. Bottomless cylinder experiment.

Calculating the Reposed Angle of the piles, its calculation formula [14] is as follows:

$$
\mathrm{a}=\arctan (2 \mathrm{H} / \mathrm{D})
$$

$\mathrm{H}$ : The apparent height of the granules, $\mathrm{cm}$

D: The apparent diameter at the bottom of the granule, $\mathrm{cm}$ Because of the peak phenomenon and inertial effect in the falling process, there are systematic errors in the calculation of the Reposed Angle using the formula. In order to minimize errors and ensure accuracy, the height $\mathrm{H}$ and the bottom diameter $\mathrm{D}$ of the broken stone pile need to be measured several times, and gaining the average, repeated experiments are carried out several times for the particle pile corresponding to each shape, the Reposed Angle is showed in Figure 6.

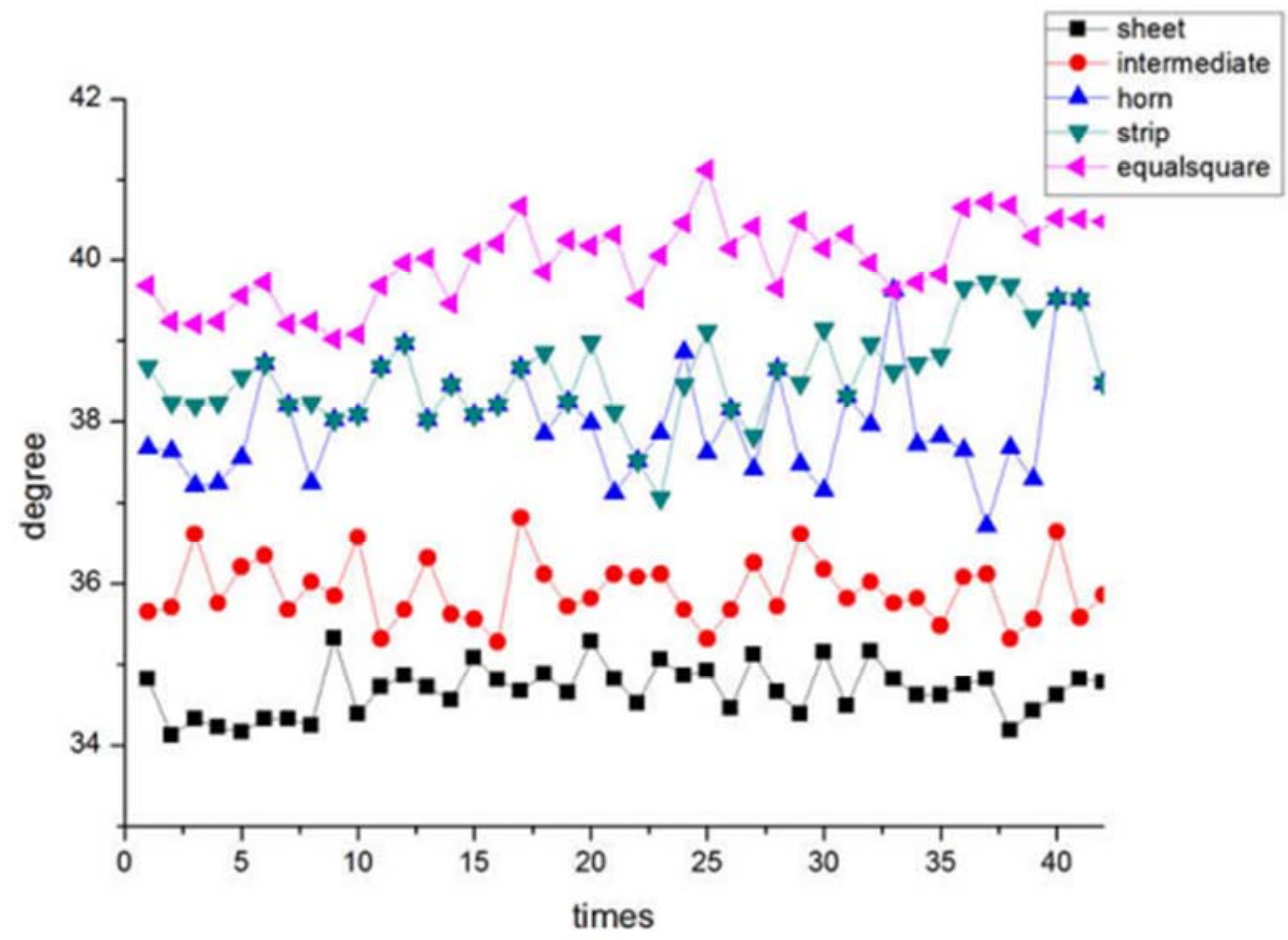

Figure 6. The changes of the Reposed Angle for corresponding gravel piles.

\subsection{Data Normality Test}

There is some error in manual operation, the stability of the experimental process needs to be verified. Firstly, the 
experimental data should be tested normally. The normal distribution of samples is the basis of the application of SPC method. The experimental data is not reliable if not conform, the normal test results of each group of data are shown in Figure 7.

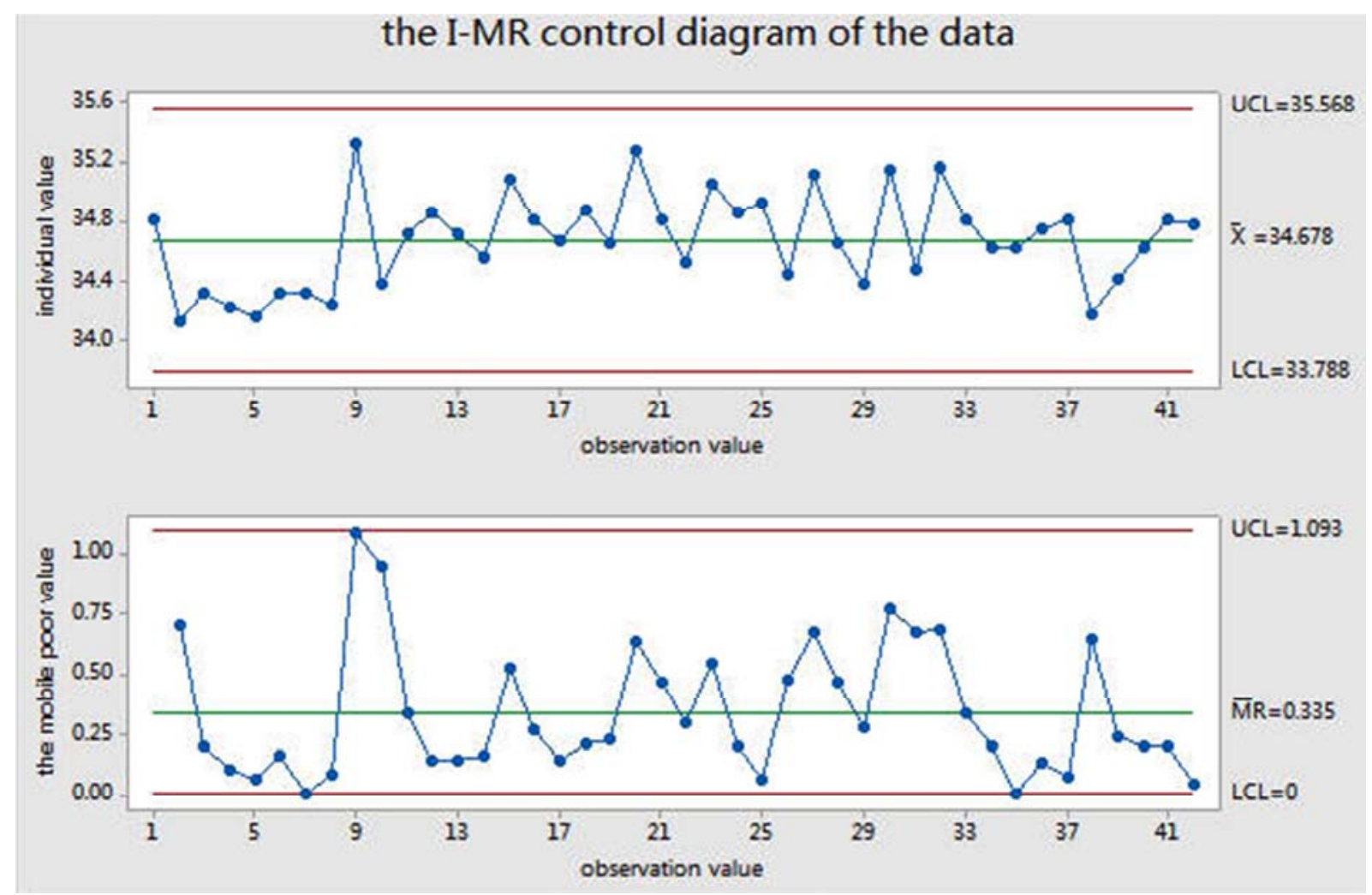

(a) The normality of sheet shape

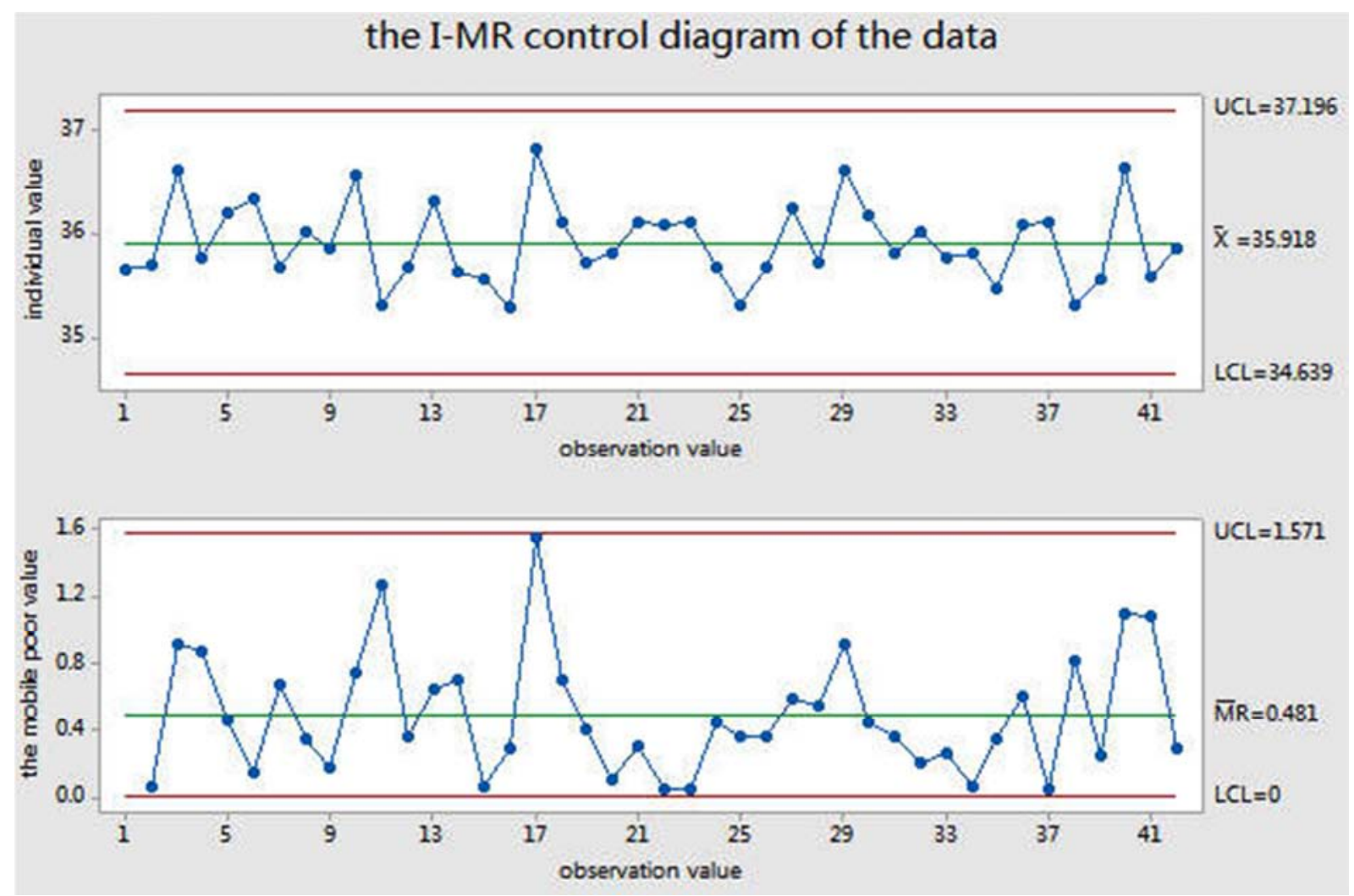

(b) The normality of intermediate shape 


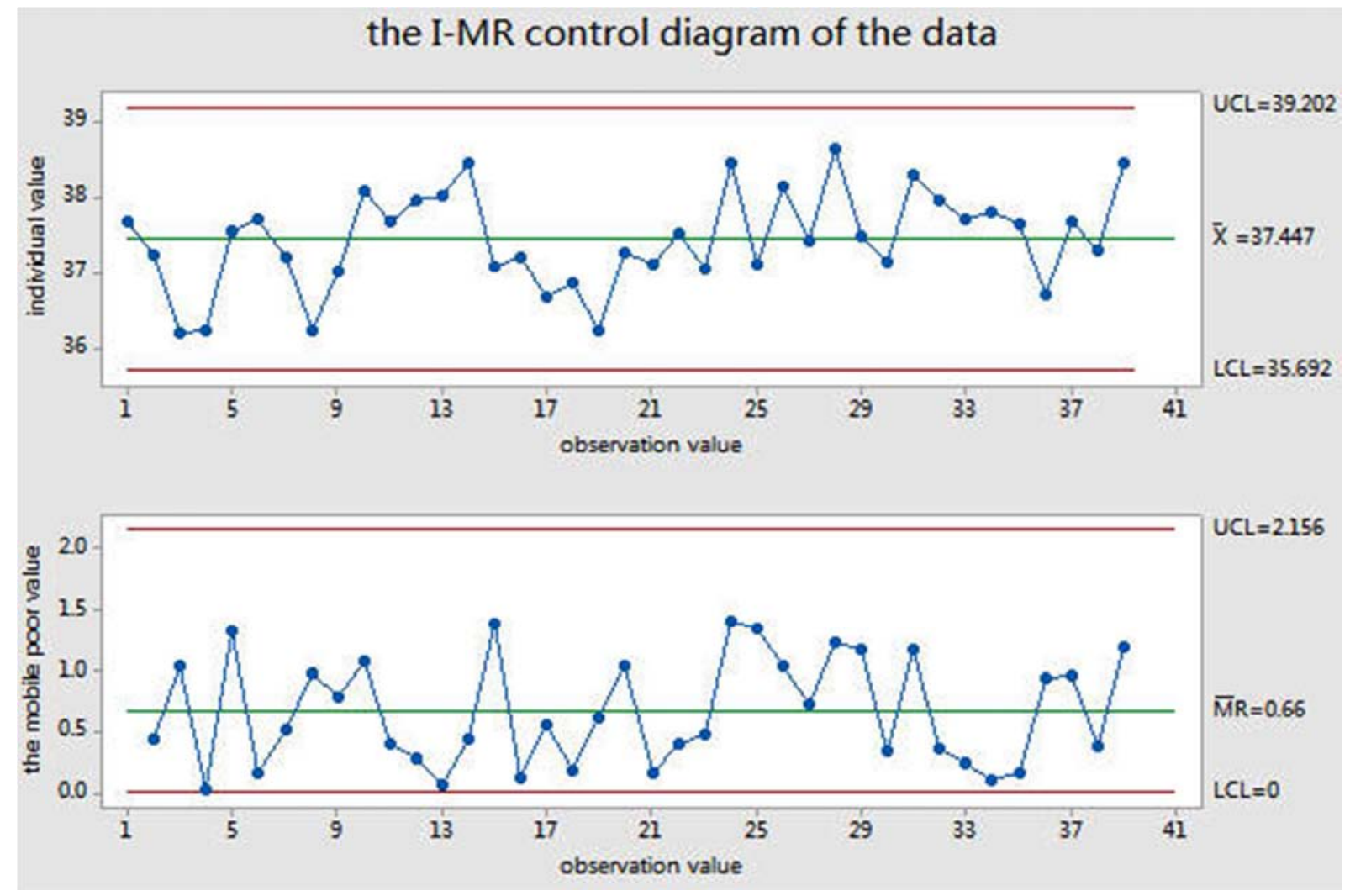

(c) The normality of horn shape

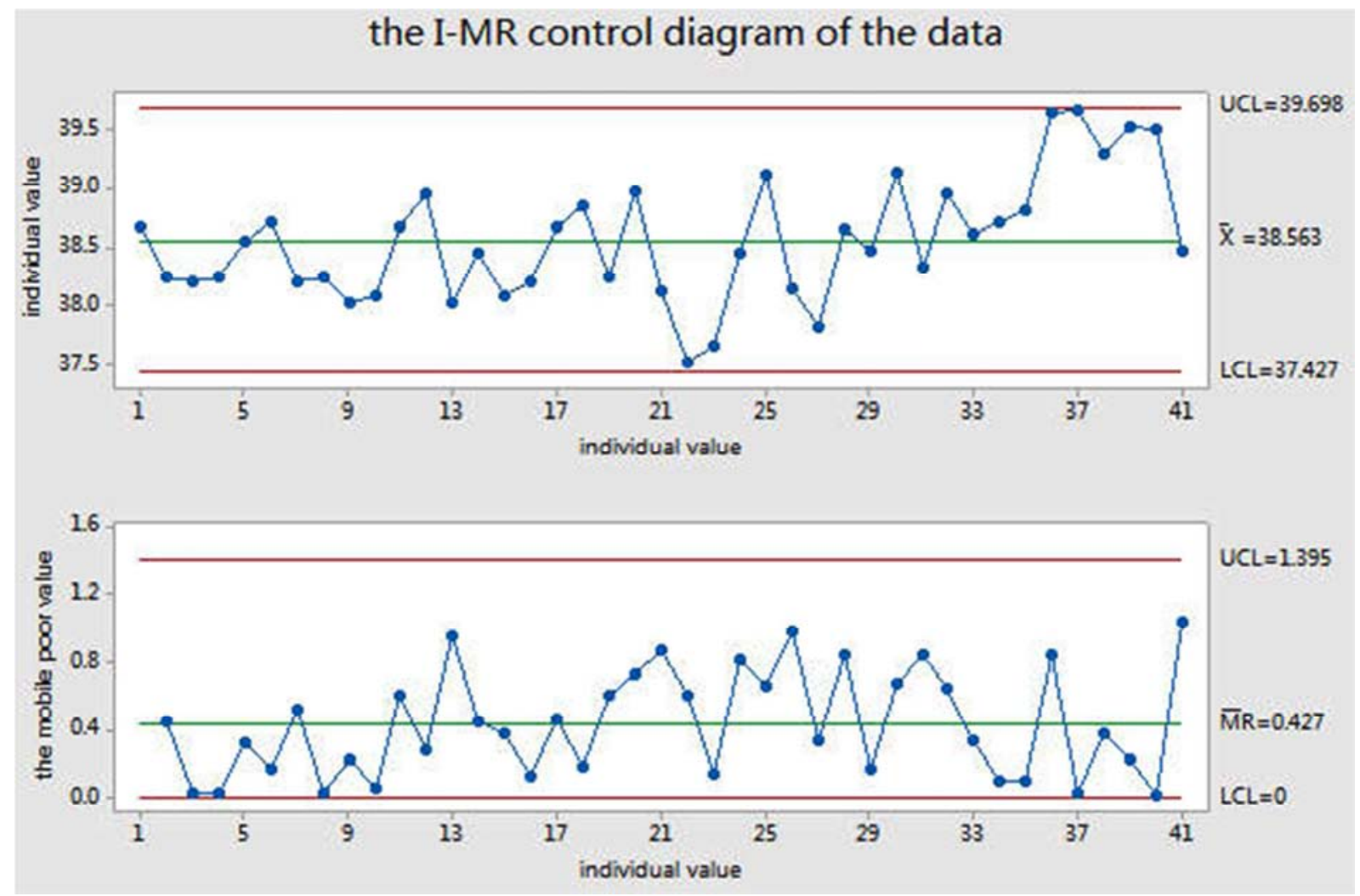

(d) The normality of strip shape 


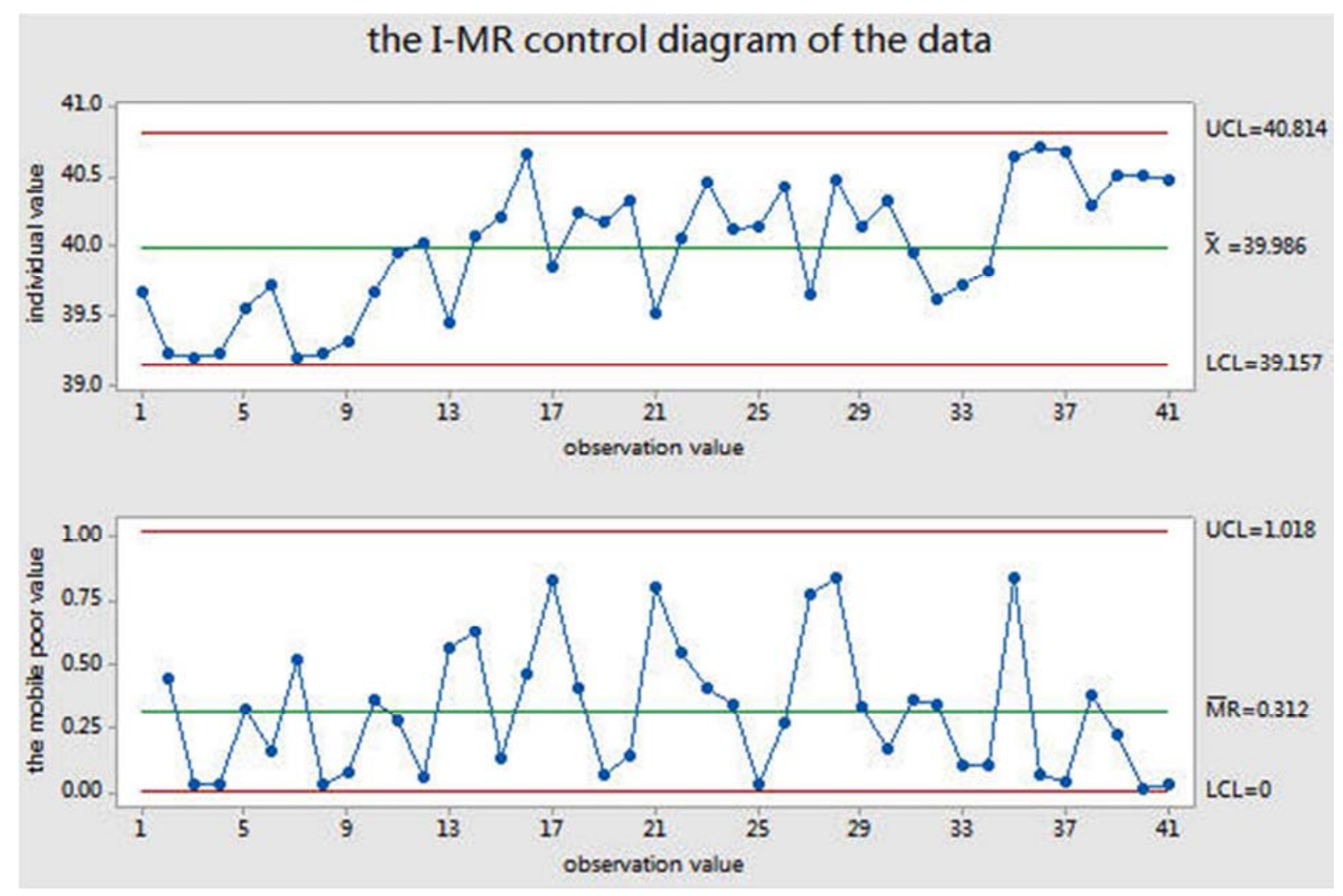

(e) The normality of equal square shape

Figure 7. The normality of the Reposed Angle of each group.

The $\mathrm{P}$ value represents the probability of normal distribution in Figure 7, the sizes are 0.514, 0.097, 0.600, 0.255 and 0.165 respectively, all are greater than 0.05 , so that each set of data satisfies the normal distribution, the experimental data are reliable.

\subsection{SPC Method and Data Control Diagram}

\subsubsection{SPC Medthod}

SPC (Statistical Process Control) statistical process control method [15] was first proposed by American scholar Shehart, mainly used for improving the quality management level, mainly for measuring, monitoring and controlling the design process. It is a scientific Data-centric quality analysis tools, applying data statistics principles, detecting, collecting and analyzing relevant data, ensuring that the experimental process is in a state of monitoring, and controlling the production process, reducing product quality variations, and pre-controlling product data obtained during production. Within a certain control limit, it helps companies to control and analyze the production process to achieve the purpose of prior control processes and improve product quality.

The SPC method considers that any process has fluctuations. It is generally divided into two forms: normal fluctuation and abnormal fluctuation. When analyzing the production process, if abnormal fluctuations occur in the production process, it is necessary to take relevant measures to eliminate the anomaly and stabilize the process. Prevent the entire process of production. Because the fluctuation of production process has statistical regularity, it believes that the production process is affected by random factors and systemic factors, based on feedback information and system factors. When only random factors affect the production process, the production process is only subject to randomness. The statistical control state of the factor influence, at this time the production process characteristics obey a stable random distribution; when only the system factors affect the production process, it indicates that the production process is in a state of statistical out of control, and the distribution characteristics of the production process will change. SPC uses this characterization to control the entire process. It emphasizes that the entire production process is controllable and functioning properly, so that the product can meet the customer's needs with high quality. The use of the SPC method is generally divided into two steps: one is to use the SPC method to draw the relevant control chart to analyze the production process. If the fluctuation range is exceeded, relevant measures can be taken to eliminate the impact; the second is to use the control chart to monitor the entire production process.

\subsubsection{Data Control Diagram}

SPC method is applied to draw the data single value I-MR control chart of each group in Minitab, as shown in Figure 8. 


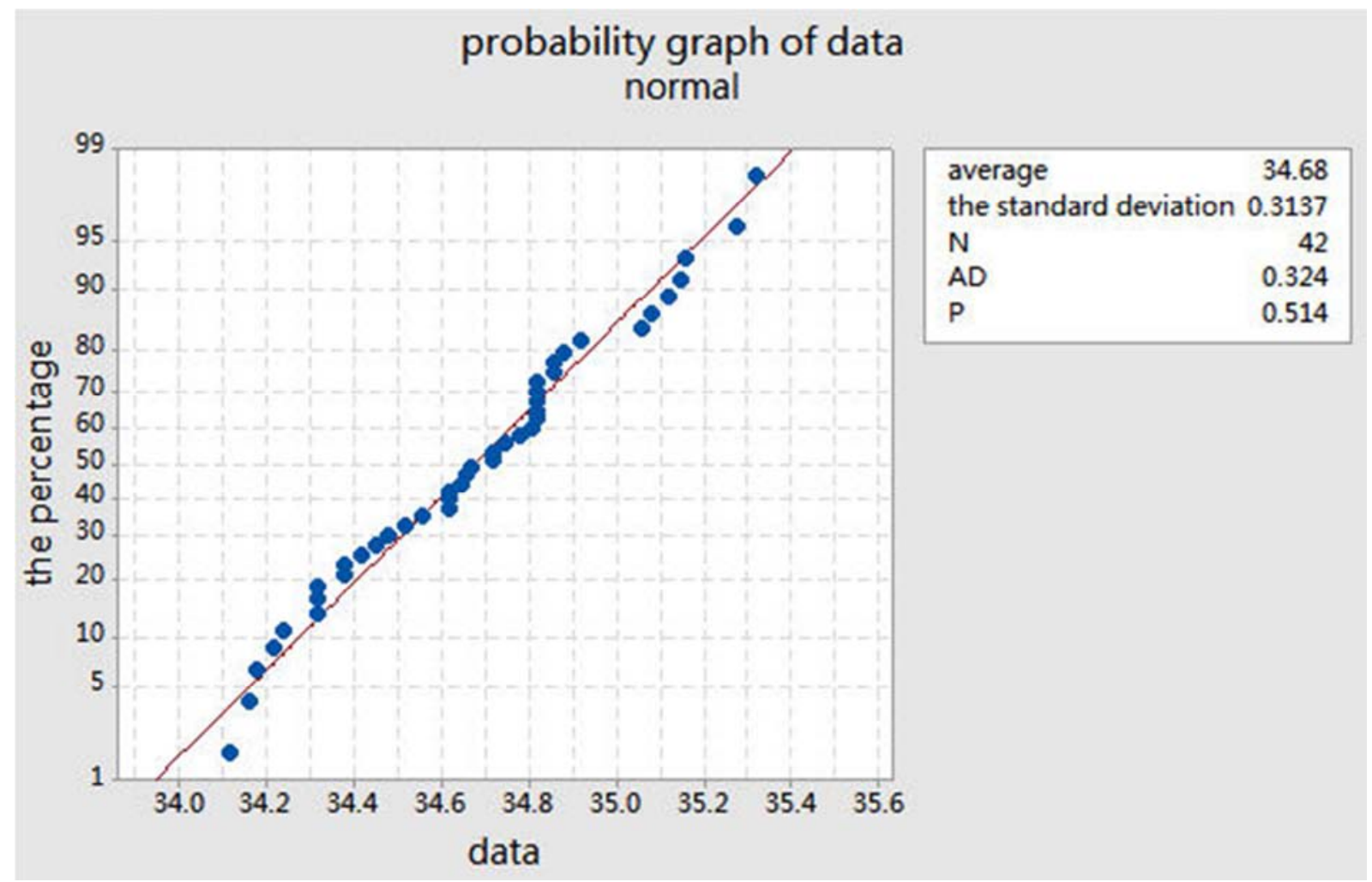

The control chart of sheet I-MR

\section{probability graph of data} normal

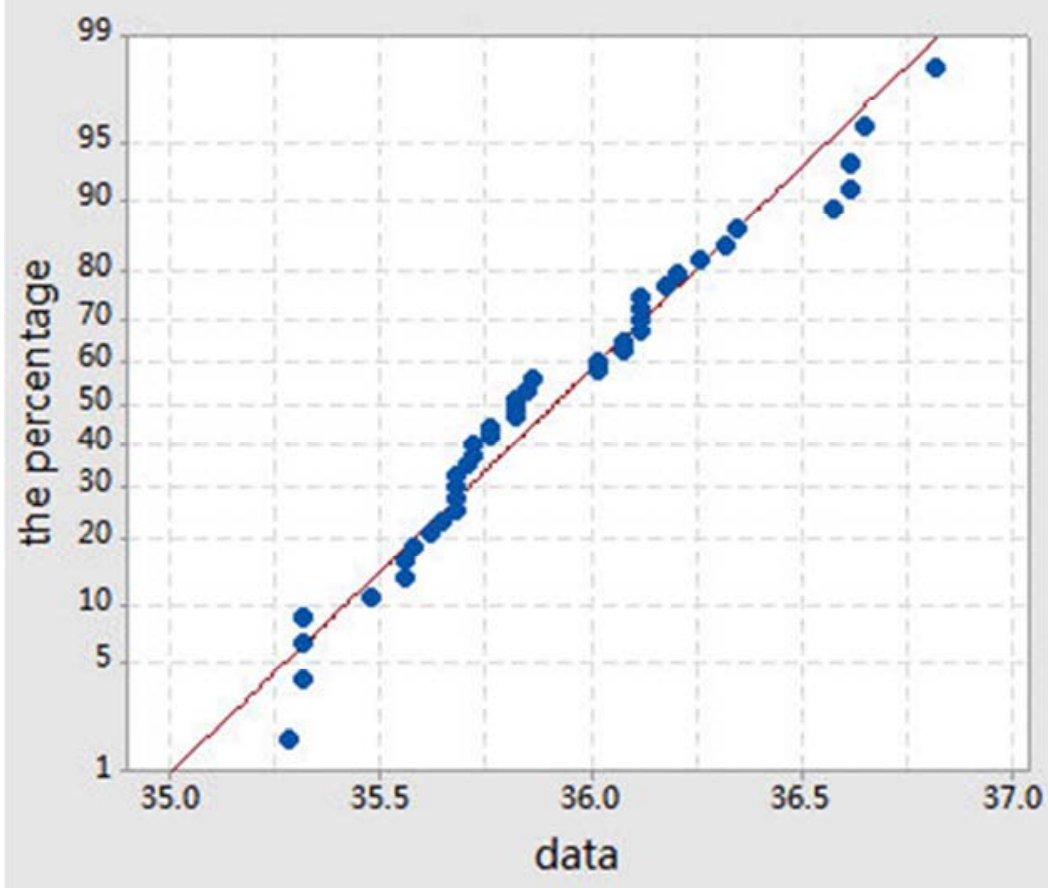

average 35.92

the standard deviation 0.3910

$\mathrm{N}$

42

$A D$

0.625

$P$

0.097

The control chart of intermediate I-MR 


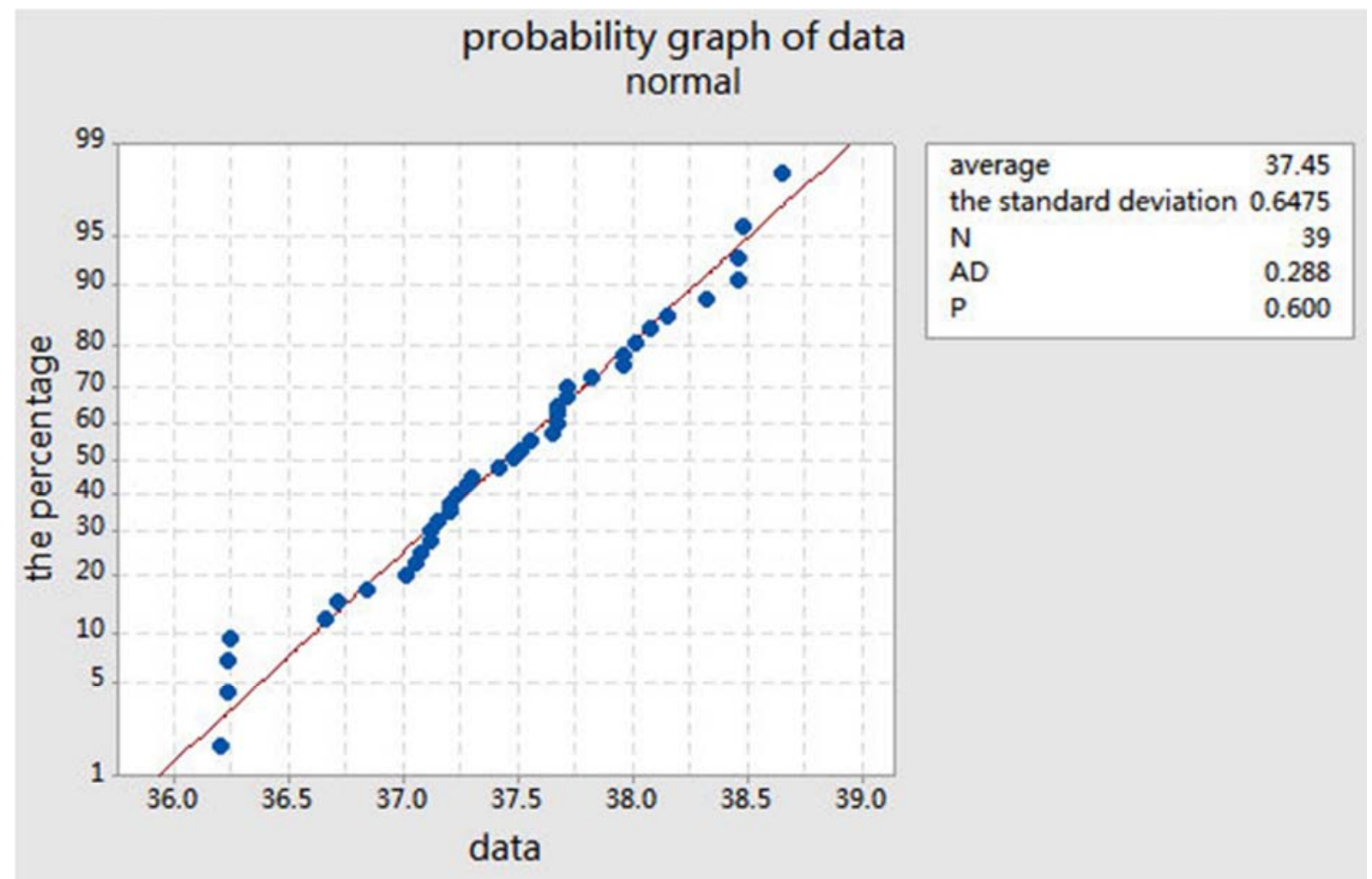

The control chart of horn I-MR

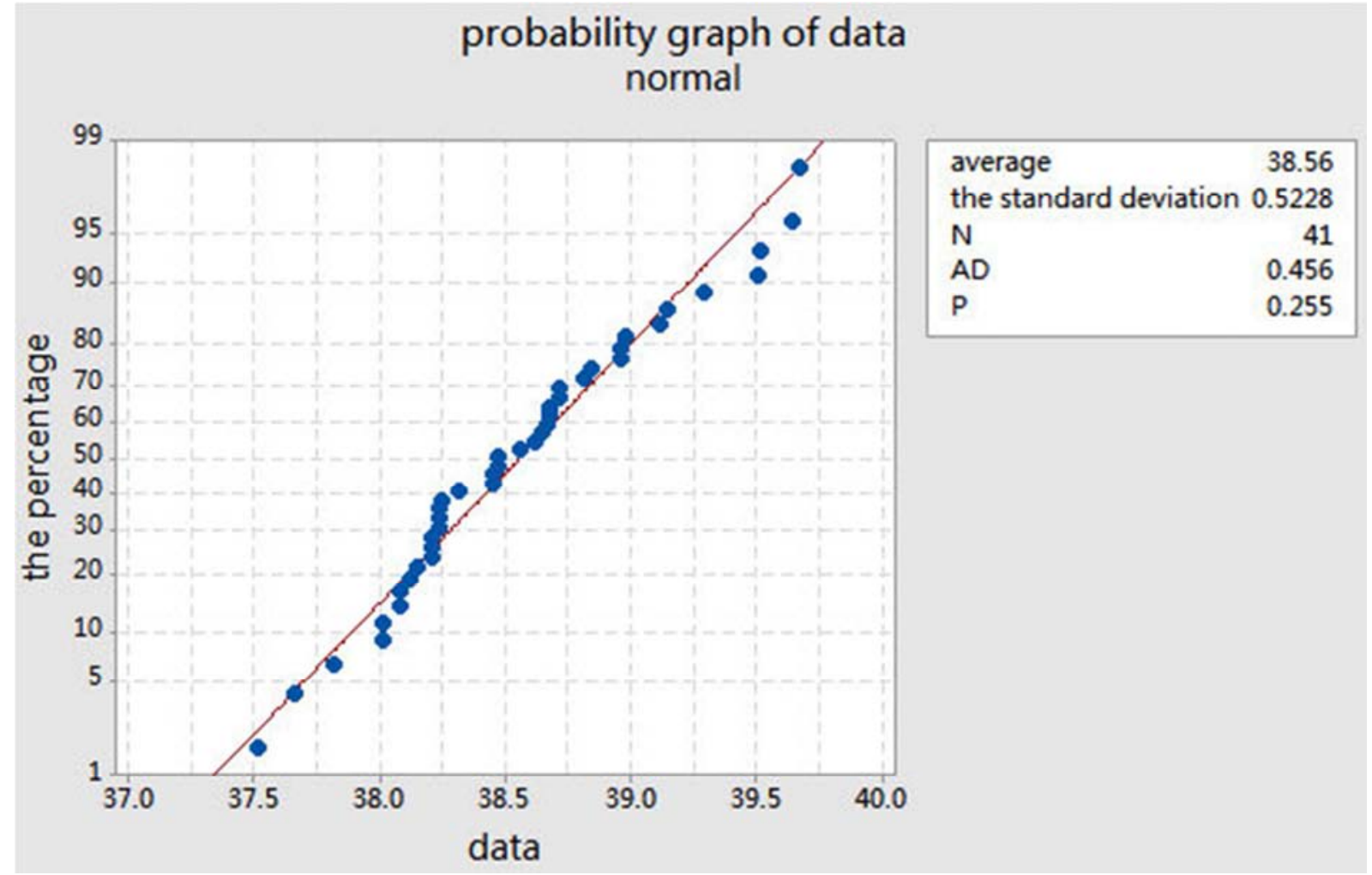

The control chart of strip I-MR 


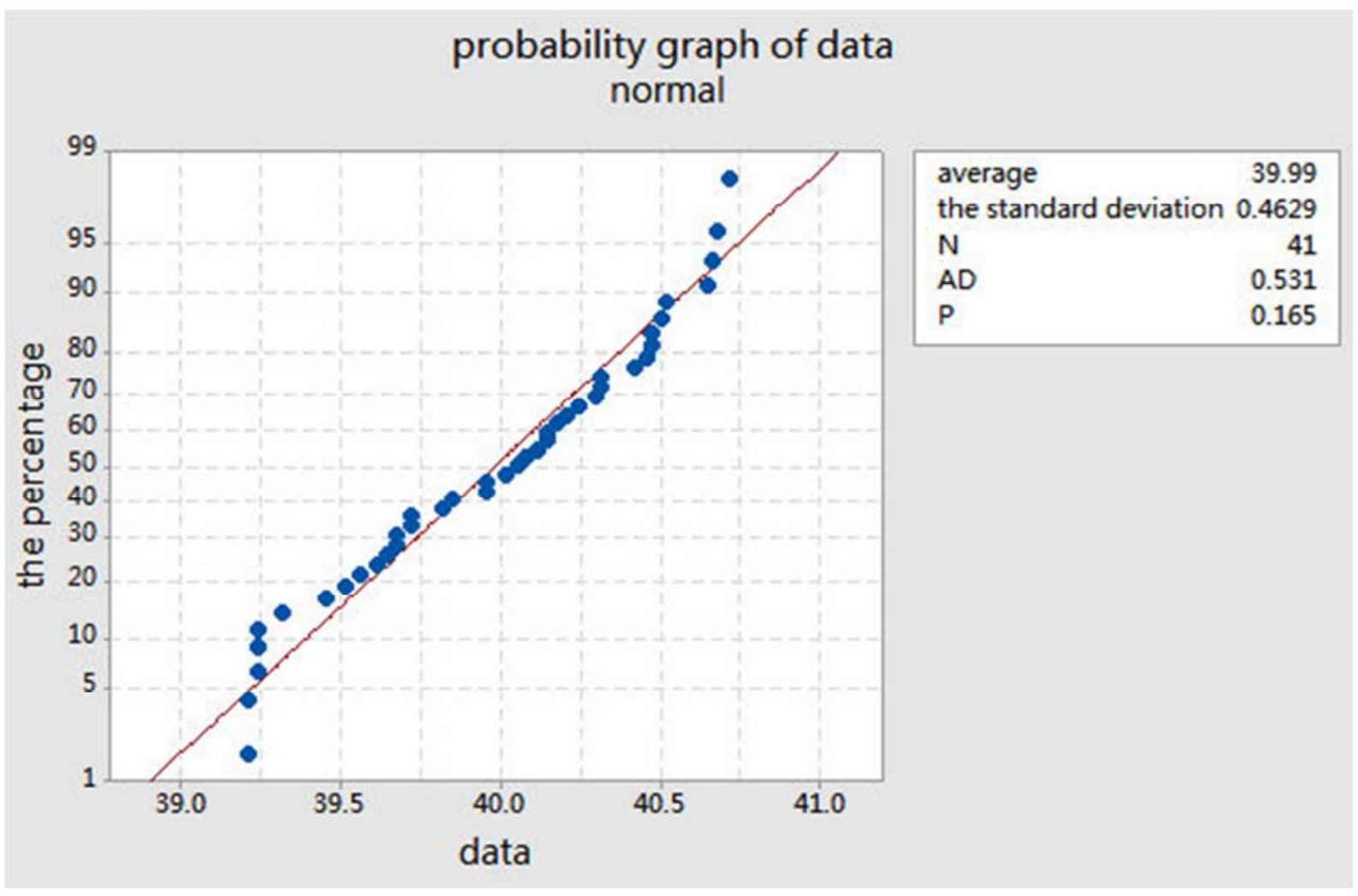

The control chart of equal square I-MR

Figure 8. The I-MR control chart of each group.

The I-MR control chart are shown in Figure 8, getting rid of a couple of exceptions, the values are all floating in the upper and lower limits. The experimental process is stable and the data is reliable, the Reposed Angle is showed for each group, As showed in Table 4.

Table 4. The Reposed Angle for Each group.

\begin{tabular}{lllll}
\hline Sheet $\left(^{()}\right)$ & Intermediate $\left(^{0}\right)$ & Horn $\left(^{0}\right)$ & Strip $\left(^{(0)}\right.$ & Equal square $\left(^{(}\right)$ \\
\hline 34.68 & 35.92 & 37.45 & 38.56 & 39.99 \\
\hline
\end{tabular}

\section{Conclusion}

This paper mainly use discrete element method for the angle of repose of different geometries numerical experiments. The results showed that the Reposed Angle of sheet, intermediate, horn, strip, equal square is $34.42^{\circ}, 35.56^{\circ}$, $37.44^{\circ}, 38.66^{\circ}, 39.56^{\circ}$ and the angle of repose is an ever-increasing process, so guessing whether the size of the angle of repose is related to the complexity of the particles. Then use bottomless cylinder experiment device to prove, test the stability of the experimental process by SPC method because there is some error in manual operation, the $\mathrm{P}$ value represents the probability of normal distribution in Figure 7, the sizes are 0.514, 0.097, 0.600, 0.255 and 0.165 respectively, all are greater than 0.05 , so that each set of data satisfies the normal distribution, the experimental data are reliable, and the I-MR control chart are shown in Figure 8, getting rid of a couple of exceptions, the values are all floating in the upper and lower limits, the experimental process is stable and the data is reliable. The results showed that the Reposed Angle of sheet, intermediate, horn, strip, equal square is $34.68^{\circ}, 35.92^{\circ}, 37.45^{\circ}, 38.56^{\circ}, 39.99^{\circ}$, the results of simulation and experiment are basically the same and the size of the angle of repose is also an increasing process. We can verify the previous guess: The size of the angle of repose is related to the complexity of the particles and the angle of repose increases as the complexity of the particles increases.

In combination with the further comparison between simulation and experiment. The results showed that the size of the Reposed Angle becomes bigger for sheet, intermediate, horn, strip, equal square for five shape types, the mechanism is that the more the particles are in contact, the more the interaction and occlusion relationship reflects between particles accurately, the more the particles are self-locked in the force chain easily, the harder it is to separate particles, the more stacking characteristics are stable. This study can provide theoretical guidance for the study of material stacking stability and related practice. 


\section{Acknowledgements}

This work is supported by Guangxi Science and Technology Development Program Funded Projects: The construction of the comprehensive test platform about earth-moving machinery operation process (No. GuiKe 1598021-2).

\section{References}

[1] SHI Q F, YAN X Q, HOU M Y, et al. Experimental study of segregation patterns in binary granular mixtures under vertical vibration [J]. Chinese Science Bulletin, 2003, 48 (7): 627-627.

[2] Ji Shun ying. Class solid - liquid phase change behavior and its constitutive equation of heterogeneous granular media [J]. Journal of mechanics, 2007, 39 (2): 223-237.

[3] Wang jian guo, Wang wei, Zhao xiaotong. Mathematical analysis of the process of simulating the natural accumulation of particles by computer [J]. Journal of north China electric power university, 1999, 26 (4): 19-22.

[4] Li Chang ning. A Study on the Relationship Between the Unhomogeneity and the Drawing-out Repose Angle of Fragmented Ore/Rock Bulk [J]. The research and development of Mining, 2002, 22 (2): 11-13.

[5] Li Yan jie. The accumulation problems of Discrete element modeling [D]: [the thesis of master]. Beijing: the university of China agricultural, 2004.

[6] Hu Guo ming. System simulation of discrete element method for particle system [M]. Wuhan: Wuhan Science University of Technology Press, 2010.
[7] Wang Guo qiang, Hao Wan jun. Discrete element method and its practice on EDEM [M]. Northwestern Polytechnical University Press, 2010.

[8] FAVIER J. Industrial Application of DEM: Opportunities and Challenges [C]. DEMOT, Brisbane Australia, 2007.

[9] Yang Yang, Tang Shougao. Selection methods on damping coefficient, stiffness coefficient and time step in granular distinct element method [J]. Computer aided engineering, 2007, 16 (3): $65 \sim 68$.

[10] Yan Ying, Ji Shun jing. Numerical simulation of the combined particle element in the experiment of shearing experiments $[\mathrm{J}]$. Journal of applied mechanics, 2009, 26 (1): $1 \sim 7$.

[11] Lleleji K. E, Zhou B. The angle of repose of bulk corn stover particles [J]. Powder Technology, 2008, 187 (2): 110-118

[12] Li Qin Liang. Study on DEM simulation of particle accumulation and bulk material transfer [D]. Shenyang: Northeastern University, 2010.

[13] Wu Ai Xiang, Sun Ye Zhi, Liu Xiang Ping. Theory of discrete body dynamics and its application [M]. Beijing: Metallurgical Industry Press, 2002.

[14] John-Paul Latham, Antonio Munjiza, Xavier Garcia, et al. Three dimension particle shape acquisiton and use of shape library for DEM an use of shape library for DEM and FEM/DEM simulation [J]. Minerals Engineering. 2008. 21 (11): 797-805.

[15] Liu wan feng, xu wu bing. Measurement of rolling friction coefficient and EDEM simulation analysis [J]. Machinery Design and Manufacture, 2018, 9 (9): 132 135. 